\title{
CORRECTION
}

\section{Correction to: Distinction of volatile flavor profiles in various skim milk products via HS-SPME-GC-MS and E-nose}

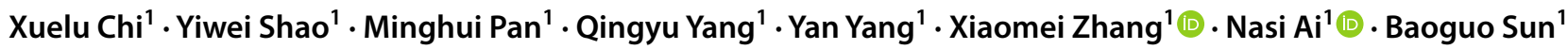

Published online: 16 April 2021

○ The Author(s), under exclusive licence to Springer-Verlag GmbH Germany, part of Springer Nature 2021

\section{Correction to: European Food Research and Technology https://doi.org/10.1007/s00217-021-03730-0}

In the original publication of the article, Fig. 1 and Table 2 has been published with an error. Also, text has been published with error in few places.

The correct Fig. 1 and Table 2 provided below in this correction.

The original article has been updated with the corrections.

Publisher's Note Springer Nature remains neutral with regard to jurisdictional claims in published maps and institutional affiliations.

The original article can be found online at https://doi.org/10.1007/ s00217-021-03730-0.

Xiaomei Zhang

zxmwaiting@163.com

$\triangle$ Nasi Ai

ainasi@btbu.edu.cn

$1 \quad$ Beijing Advanced Innovation Center for Food Nutrition and Human Health, Beijing Engineering and Technology Research Center of Food Additives, Beijing Higher Institution Engineering Research Center of Food Additives and Ingredients, Beijing Technology and Business

University, Beijing 100048, China 


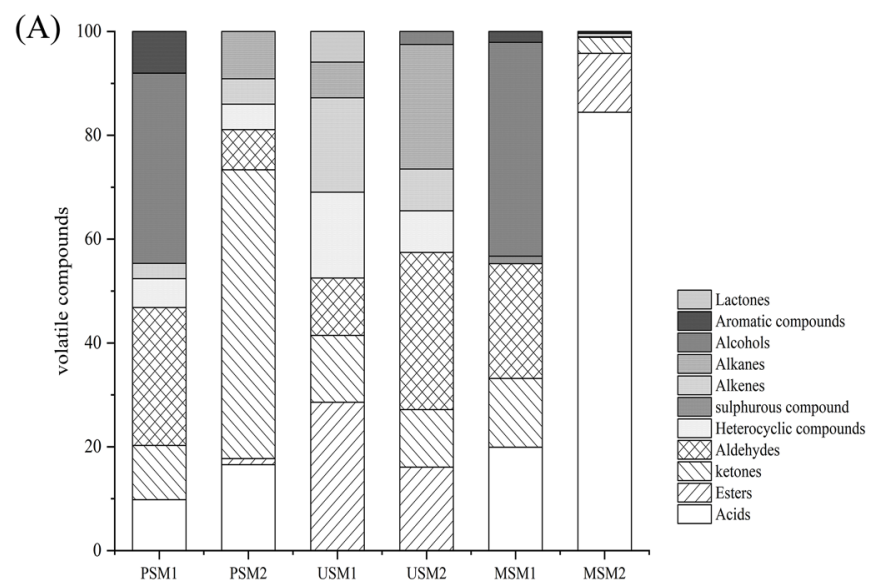

(B)
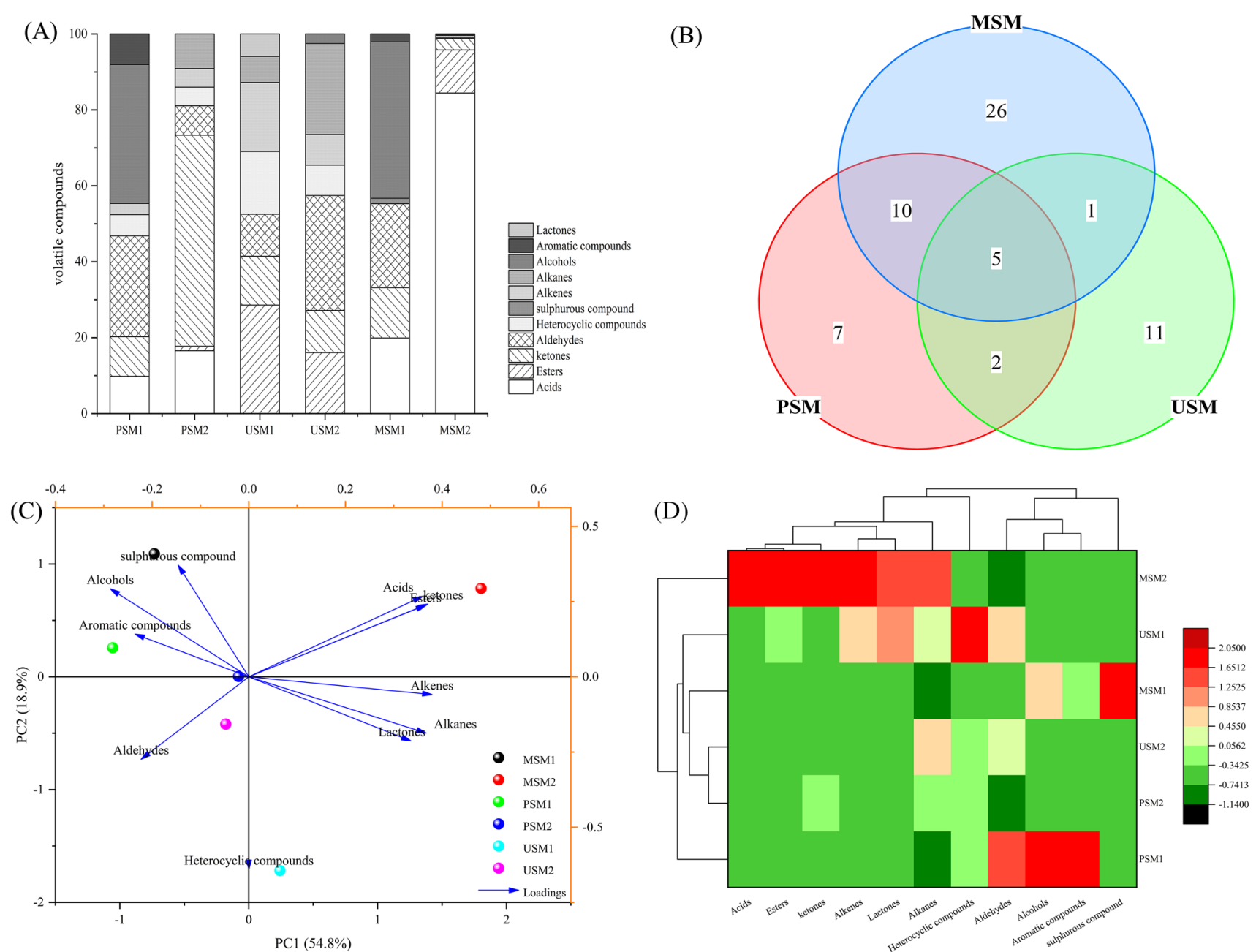

Fig. 1 Statistical analysis of the volatile compounds in skim milk samples. a Content ratios in types of volatile compounds; $\mathbf{b}$ Venn diagram analysis of the samples; c classification by PCA of the samples; d cluster heatmap analysis upon the classification of the samples and

volatile compounds. PSM pasteurized skim milk, USM ultra-hightemperature skim milk, MSM modified skim milk by lipase-catalyzed process 
Table 2 Volatile compounds in skim milk samples identified by HS-SPME-GC-MS

\begin{tabular}{|c|c|c|c|c|c|c|c|c|}
\hline \multirow[t]{2}{*}{ Compound name } & \multirow[t]{2}{*}{ RI } & \multirow[t]{2}{*}{ CAS } & \multicolumn{6}{|c|}{ Content $(\mu \mathrm{g} / \mathrm{L})$} \\
\hline & & & PSM1 & PSM2 & USM1 & USM2 & MSM1 & MSM2 \\
\hline \multicolumn{9}{|l|}{ Acids } \\
\hline 9-Decenoic acid & 1360 & $14436-32-9$ & - & - & - & - & - & $3.36 \pm 0.63$ \\
\hline Butanoic acid & 792 & $107-92-6$ & - & - & - & - & - & $30.94 \pm 2.77$ \\
\hline Dodecanoic acid & 1568 & $143-07-7$ & - & - & - & - & - & $29.39 \pm 1.84$ \\
\hline Heptanoic acid & 1085 & $111-14-8$ & - & - & - & - & - & $3.90 \pm 0.04$ \\
\hline Hexanoic acid & 984 & $142-62-1$ & $0.36 \pm 0.07$ & $0.20 \pm 0.10$ & - & - & $0.37 \pm 0.05$ & $414.22 \pm 33.95$ \\
\hline$n$-Decanoic acid & 1365 & $334-48-5$ & $0.39 \pm 0.08$ & $0.60 \pm 0.24$ & - & - & $0.53 \pm 0.00$ & $186.45 \pm 3.61$ \\
\hline Octanoic acid & 1175 & $124-07-2$ & $0.62 \pm 0.16$ & $1.35 \pm 0.46$ & - & - & $0.59 \pm 0.10$ & $369.83 \pm 6.83$ \\
\hline Tetradecanoic acid & 1767 & $544-63-8$ & - & - & - & - & - & $3.87 \pm 2.84$ \\
\hline Undecanoic acid & 1436 & $112-37-8$ & - & - & - & - & - & $1.33 \pm 0.02$ \\
\hline \multicolumn{9}{|l|}{ Alcohols } \\
\hline 1-Decanol, 2-hexyl- & 1810 & $2425-77-6$ & - & - & - & $0.20 \pm 0.06$ & - & - \\
\hline 1-Octanol & 1037 & $111-87-5$ & $0.19 \pm 0.02$ & - & - & - & $0.21 \pm 0.10$ & - \\
\hline 1-Octen-3-ol & 982 & $3391-86-4$ & $0.19 \pm 0.02$ & - & - & - & $0.18 \pm 0.05$ & - \\
\hline 2-Ethylhexanol & 1034 & $104-76-7$ & $4.73 \pm 0.58$ & - & - & - & $2.71 \pm 0.39$ & - \\
\hline \multicolumn{9}{|l|}{ Aldehydes } \\
\hline (Z)-2-Nonenal & 963 & $60784-31-8$ & $0.07 \pm 0.00$ & - & - & - & $0.29 \pm 0.05$ & - \\
\hline Decanal & 1206 & $112-31-2$ & - & $0.33 \pm 0.12$ & $0.72 \pm 0.05$ & $0.67 \pm 0.02$ & - & - \\
\hline Nonanal & 1105 & $124-19-6$ & $2.54 \pm 0.30$ & $0.68 \pm 0.26$ & $2.01 \pm 0.24$ & $1.82 \pm 0.33$ & $1.23 \pm 1.22$ & $1.20 \pm 0.12$ \\
\hline Octanal & 1002 & $124-13-0$ & $0.29 \pm 0.05$ & - & - & - & $0.14 \pm 0.03$ & - \\
\hline \multicolumn{9}{|l|}{ Alkanes } \\
\hline Decane & 1059 & $124-18-5$ & - & - & $0.43 \pm 0.07$ & - & - & - \\
\hline Dodecane & 1199 & $112-40-3$ & - & $0.27 \pm 0.04$ & $0.75 \pm 0.26$ & $0.43 \pm 0.04$ & - & - \\
\hline Eicosane & 1800 & $112-95-8$ & - & - & $0.30 \pm 0.02$ & - & - & - \\
\hline Heptadecane & 1700 & $629-78-7$ & - & - & - & $0.26 \pm 0.07$ & - & - \\
\hline Hexadecane & 1600 & $544-76-3$ & - & - & - & $0.30 \pm 0.04$ & - & - \\
\hline Nonane & 903 & $111-84-2$ & - & - & - & $0.49 \pm 0.05$ & - & - \\
\hline Octadecane & 1792 & $593-45-3$ & - & - & - & $0.21 \pm 0.07$ & - & - \\
\hline Tetradecane & 1399 & $629-59-4$ & - & $0.56 \pm 0.22$ & $0.21 \pm 0.04$ & $0.29 \pm 0.02$ & - & $1.70 \pm 0.13$ \\
\hline Tridecane & 1299 & $629-50-5$ & - & $0.36 \pm 0.14$ & - & - & - & $1.14 \pm 0.10$ \\
\hline \multicolumn{9}{|l|}{ Alkenes } \\
\hline 5-Tetradecene, $(E)$ - & 1384 & $041446-66-6$ & - & - & - & - & - & $7.16 \pm 1.14$ \\
\hline 6-Dodecene, $(E)$ - & 1139 & $68526-77-2$ & & - & $0.41 \pm 0.11$ & - & - & - \\
\hline 7-Hexadecene, $(Z)$ - & 1593 & $35507-09-6$ & - & - & - & - & - & $1.13 \pm 0.68$ \\
\hline D-Limonene & 1030 & $5989-27-5$ & $0.41 \pm 0.05$ & $0.64 \pm 0.27$ & $4.09 \pm 0.19$ & $0.66 \pm 0.05$ & - & - \\
\hline \multicolumn{9}{|l|}{ Esters } \\
\hline Butanoic acid, butyl ester & 998 & $109-21-7$ & - & $0.15 \pm 0.06$ & - & - & - & - \\
\hline Butanoic acid, ethyl ester & 807 & $105-54-4$ & - & - & - & - & - & $14.02 \pm 2.37$ \\
\hline Decanoic acid, ethyl ester & 1395 & $110-38-3$ & - & - & - & - & - & $19.83 \pm 1.08$ \\
\hline Decanoic acid, methyl ester & 1354 & $110-42-9$ & - & - & - & - & - & $0.98 \pm 0.03$ \\
\hline Dodecanoic acid, ethyl ester & 1586 & $106-33-2$ & - & - & - & - & - & $7.45 \pm 0.32$ \\
\hline Ethyl 9-tetradecenoate & 1771 & $1000336-60-8$ & - & - & - & - & - & $2.66 \pm 3.41$ \\
\hline Heptanoic acid, ethyl ester & 1096 & $106-30-9$ & - & - & - & - & - & $1.54 \pm 0.09$ \\
\hline Hexadecanoic acid, ethyl ester & 1997 & $628-97-7$ & - & - & - & - & - & $0.98 \pm 1.10$ \\
\hline $\begin{array}{l}\text { Hexanoic acid, 2-methylpro- } \\
\text { pyl ester }\end{array}$ & 1450 & $105-79-3$ & - & - & - & - & - & $2.26 \pm 0.13$ \\
\hline Hexanoic acid, ethyl ester & 994 & $123-66-0$ & - & - & - & - & - & $28.39 \pm 2.03$ \\
\hline Nonanoic acid, ethyl ester & 1305 & $123-29-5$ & - & - & - & - & - & $0.85 \pm 0.02$ \\
\hline Octanoic acid, ethyl ester & 1182 & $106-32-1$ & - & - & - & - & - & $55.79 \pm 1.66$ \\
\hline
\end{tabular}


Table 2 (continued)

\begin{tabular}{|c|c|c|c|c|c|c|c|c|}
\hline \multirow[t]{2}{*}{ Compound name } & \multirow[t]{2}{*}{ RI } & \multirow[t]{2}{*}{ CAS } & \multicolumn{6}{|c|}{ Content $(\mu \mathrm{g} / \mathrm{L})$} \\
\hline & & & PSM1 & PSM2 & USM1 & USM2 & MSM1 & MSM2 \\
\hline Octanoic acid, methyl ester & 1126 & $111-11-5$ & & - & $2.93 \pm 0.18$ & - & - & $0.95 \pm 0.04$ \\
\hline $\begin{array}{l}\text { Sulfurous acid, butyl nonyl } \\
\text { ester }\end{array}$ & 1035 & $1000309-17-6$ & - & - & - & $1.32 \pm 0.15$ & - & - \\
\hline Tetradecanoic acid, ethyl ester & 1797 & 124-06-1 & - & - & - & - & - & $1.67 \pm 0.81$ \\
\hline Triacetin & 1352 & $102-76-1$ & - & - & $4.15 \pm 0.78$ & - & - & - \\
\hline Vinyl butyrate & 1324 & $123-20-6$ & - & - & - & - & - & $2.95 \pm 0.37$ \\
\hline \multicolumn{9}{|l|}{ Ketones } \\
\hline 1-Phenyl-2-butanone & 1228 & $1007-32-5$ & - & $6.28 \pm 1.66$ & - & - & - & - \\
\hline 2-Heptanone & 894 & $110-43-0$ & - & $0.18 \pm 0.03$ & $0.85 \pm 0.04$ & - & - & $4.31 \pm 0.27$ \\
\hline 2-Nonanone & 1093 & $821-55-6$ & $0.48 \pm 0.07$ & $0.78 \pm 0.35$ & $1.65 \pm 0.05$ & $0.59 \pm 0.04$ & $0.55 \pm 0.16$ & $21.43 \pm 2.47$ \\
\hline 2-Pentadecanone & 1597 & $2345-28-0$ & - & - & - & - & - & $0.92 \pm 0.38$ \\
\hline 2-Tridecanone & 1461 & $593-08-8$ & - & - & - & - & - & $3.89 \pm 0.41$ \\
\hline 2-Undecanone & 1294 & $112-12-9$ & $0.07 \pm 0.02$ & - & $0.69 \pm 0.04$ & $0.32 \pm 0.05$ & $0.13 \pm 0.01$ & $7.41 \pm 0.58$ \\
\hline 3-Octen-2-one & 1059 & $1669-44-9$ & $0.16 \pm 0.02$ & - & - & - & - & - \\
\hline 6-Methyl-5-hepten-2-one & 991 & $110-93-0$ & $0.42 \pm 0.13$ & - & - & - & $0.16 \pm 0.09$ & - \\
\hline \multicolumn{9}{|l|}{ Lactones } \\
\hline $\begin{array}{l}\text { 2(3H)-Furanone, 5-heptyl- } \\
\text { dihydro- }\end{array}$ & 1576 & $104-67-6$ & - & - & - & - & - & $0.82 \pm 0.15$ \\
\hline $\begin{array}{l}\text { 2(3H)-Furanone, dihydro- } \\
5 \text {-pentyl- }\end{array}$ & 1367 & 104-61-0 & - & - & $1.46 \pm 0.11$ & - & - & - \\
\hline $\begin{array}{l}2 H \text {-Pyran-2-one, 6-hexyltet- } \\
\text { rahydro- }\end{array}$ & 1681 & $710-04-3$ & - & - & - & - & - & $0.81 \pm 0.25$ \\
\hline \multicolumn{9}{|l|}{ Aromatic compounds } \\
\hline Acetophenone & 1058 & $98-86-2$ & $0.32 \pm 0.03$ & - & - & - & $0.16 \pm 0.01$ & - \\
\hline Benzaldehyde & 965 & $100-52-7$ & $0.8 \pm 0.08$ & - & - & - & - & - \\
\hline Heterocyclic compound & & & & & & & & - \\
\hline 2-Pentyl-furan & 992 & $3777-69-3$ & $0.77 \pm 0.55$ & $0.64 \pm 0.38$ & $4.09 \pm 3.95$ & $0.66 \pm 0.55$ & - & - \\
\hline \multicolumn{9}{|l|}{ Sulphurous compound } \\
\hline Dimethyl sulfone & 925 & $67-71-0$ & - & - & - & - & $0.11 \pm 0.00$ & - \\
\hline Total & & & $12.41 \pm 1.74$ & $13.02 \pm 1.73$ & $24.76 \pm 2.23$ & $8.23 \pm 1.00$ & $7.35 \pm 2.07$ & $1235.53 \pm 39.57$ \\
\hline
\end{tabular}

